\title{
The Study on PM Emission Characteristics of Light-duty Diesel Vehicle Equipped with DOC+POC
}

\author{
Wang Ji-guang ${ }^{1, a}$, Fang Xi-yu ${ }^{1, b}$, Zhang Shi-min ${ }^{1, c}$, Shen shu ${ }^{1, d}$ \\ ${ }^{1}$ China Automotive Technology \& Research Center \\ a wangjiguang@catarc.ac.cn, ${ }^{b}$ fangxiyu@catarc.ac.cn, ${ }^{c}$ zhangshimin@catarc.ac.cn, \\ dshenshu@catarc.ac.cn
}

\begin{abstract}
Keywords: PEMS, Light-duty Diesel Vehicle, Particulate Oxidation Catalyst, Filtration Efficiency, Number Concentration, Mass Concentration

Abstract. Using Portable Emission Measurement System (PEMS in short), the PM and gaseous pollutants emission in China III light-duty diesel vehicle before and after installing DOC + POC under cold and hot start conditions are studied respectively. Meanwhile, the POC filtration efficiency and size distribution of both particle number concentration and mass concentration are analyzed. The result show the PM emission from vehicle installed POC can meet China IV standard, and the filtration efficiency of both particle number and mass is $25 \% \sim 55 \%$ under cold and hot start. POC filtration efficiency under cold start is $10 \%$ higher than hot start. However, the filtration efficiency is different under different size distribution. PM is generated mainly in acceleration process.
\end{abstract}

\section{Introduction}

As a new type of catalyst optimized for reducing PM and PN emission, POC has been applied in part of China IV light-duty diesel vehicles. Due to the higher operating temperature required by the POC, it needs to be used in conjunction with DOC. Compared with DPF, POC has the advantages of low cost, no need of complex calibration process, short development period and good reliability, while avoiding the adverse effect of high sulfur fuel on the catalyst ${ }^{[1]}$. In addition to the application in new light-duty diesel vehicles, domestic and foreign enterprises and research institutions have carried out some practical research for DOC+POC applied in in-use diesel-duty vehicles and evaluateing effect of emission reduction.In this paper, selecting a China Stage III light-duty diesel vehicles as the object of study, in-depth study the original vehicle emissions and installation of POC before and after the end of the concentration of pollutants change under cold strat and hot start , exploring the filtration efficiency and effect of POC in the use of diesel vehicles emissions control.

\section{Experimental Program}

\section{Test Vehicle}

The specific parameters of Test Vehicle and DOC+POC are listed in Table 1. Test fuel for 40ppmS low sulfur vehicle diesel. 
Table1 Specific parameters of Test Vehicle and DOC + POC

\begin{tabular}{l|l}
\hline \multicolumn{1}{c|}{ Content } & \multicolumn{1}{c}{ Specific parameters } \\
\hline Engine type & Four stroke straight line direct injection \\
\hline Total quality $/ \mathrm{kg}$ & 2510 \\
\hline Curb quality $/ \mathrm{kg}$ & 1790 \\
\hline Rated power $/ \mathrm{kW}$ & 110 \\
\hline Distance $/ \mathrm{km}$ & 594 \\
\hline Maximum speed $/ \mathrm{km} / \mathrm{h}$ & 180 \\
\hline Displacement $/ \mathrm{L}$ & 1.996 \\
\hline & $\emptyset 155 \times 110$, Cordierite carrier, coated with platinum \\
DOC size $/ \mathrm{mm}$ & and palladium \\
\hline POC size $/ \mathrm{mm}$ & $\emptyset 155 \times 220$, Metal carrier, no precious metals \\
\hline
\end{tabular}

\section{Test equipment}

The test is carried out on a vehicle test dynamic and tested by using a PEMS. PEMS consists of an onboard gaseous pollutant measuring instrument OBS-2200 and an onboard particle measuring instrument ELPI to test the discharge characteristics under different conditions. OBS-2200 measures the concentrations of instantaneous $\mathrm{CO}, \mathrm{CO} 2, \mathrm{NO}$ and $\mathrm{THC}$, temperature and pressure of the exhaust emissions from the vehicle, ambient temperature, humidity, and pressure. ELPI measures the mass concentration of the particles in each instantaneous particle size, number of concentration distribution ${ }^{[2]}$.

\section{Test program}

In this paper, author carry out the emission test before and after installing DOC+POC under cold start and hot start conditions (after 30min from the end of cold start test) in accordance with GB18352.3-2005. In order to reduce the test error and data fluctuations, respectively, under both of states, will take the average value of the analysis in different sampling position of the pollutant emissions after two tests. Test equipment and test location shown in Figure 1.

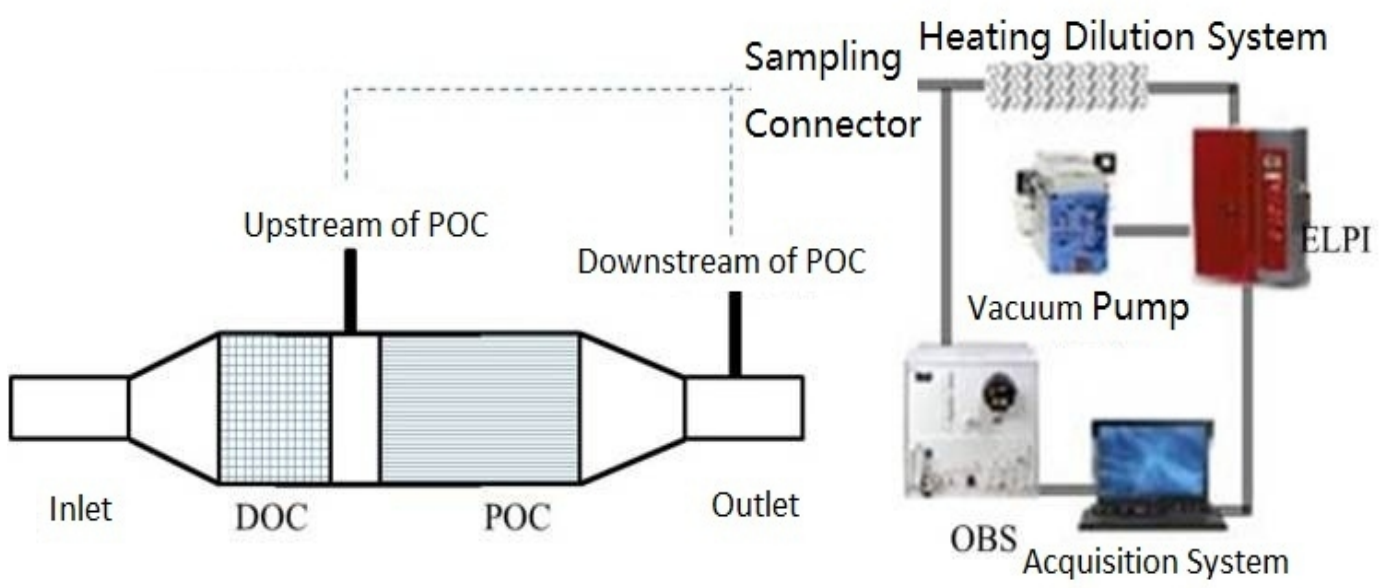

Fig.1 Test system and DOC + POC sampling position 


\section{Test results and Data analysis}

\section{Analysis of Emissions Results}

Figure 2 for the test results of original vehicle emissions and pollutant emissions after installing DOC + POC under cold start and hot start conditions.

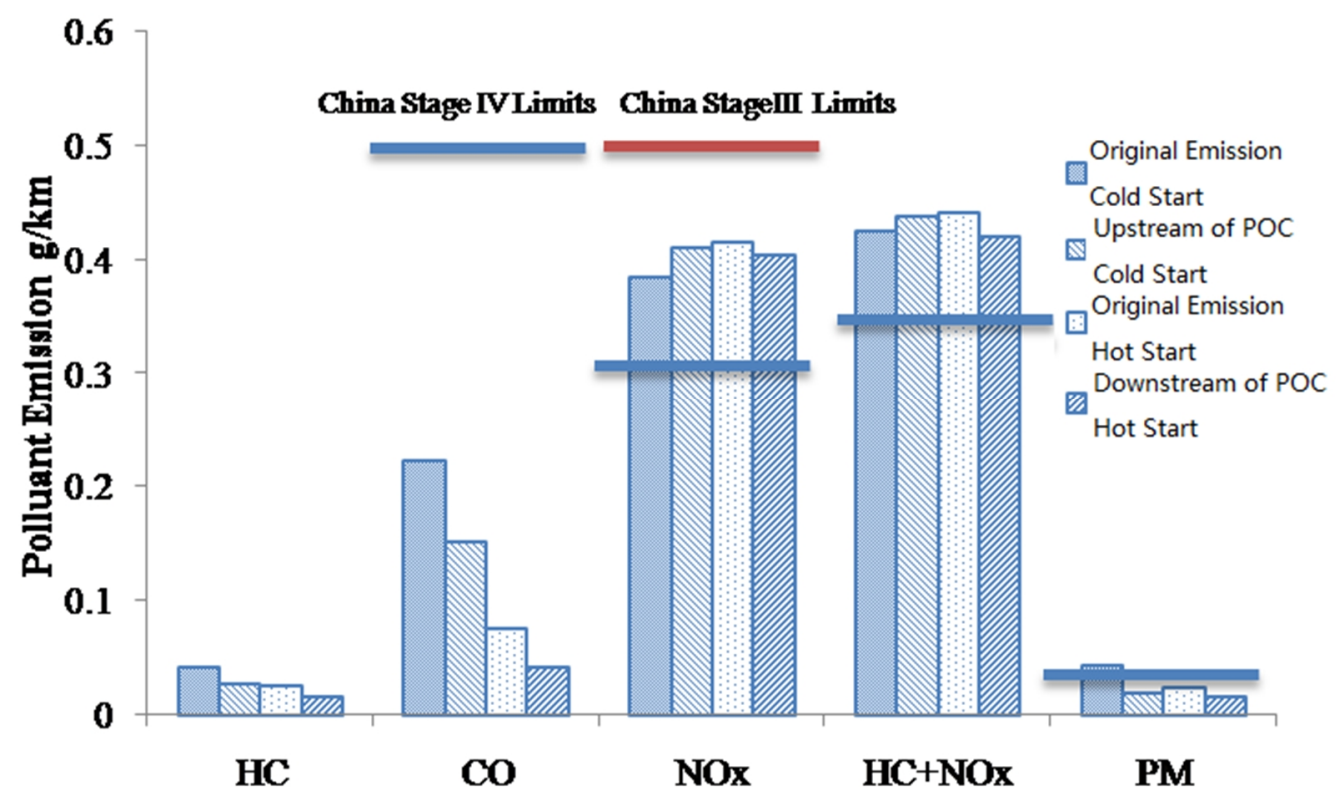

Fig.2 Original vehicle emissions and pollutant emissions after installing DOC + POC

According to the emission results and corresponding regulatory limits, the vehicle emissions after installing DOC + POC can meet the requirements of China Stage IV emission limits under the cold start and hot start. Compared with the original vehicle emissions, $\mathrm{CO}$ and $\mathrm{HC}$ emissions after installing of DOC + POC reduce about $60 \%$, the main reason is that platinum and palladium coated in DOC can oxidize CO and HC. Meanwhile, compared with cold start emissions, the $\mathrm{CO}$ and $\mathrm{HC}$ emissions under hot start conditions can reduce to $30 \%$ and $60 \%$, the main reason is that the vehicle after a period of time in the DOC carrier and catalyst temperature gradually increased, the CO and $\mathrm{HC}$ show good performance. The change of NOx and $\mathrm{HC}+\mathrm{NOx}$ emission result after installing DOC + POC was little, which indicated that the hot and cold start of the vehicle and the DOC + POC had little effect on the NOx emission, main reason is that the DOC catalyst does not play important role of precious metal rhodium in reducing NOx.

The original vehicle emissions and PM emission after installing DOC + POC can meet the requirements of China Stage IV emission limits under the hot start. Meanwhile the installation of DOC + POC PM emission value of about $45 \%$ to $60 \%$ of the original vehicle emissions under hot and cold start, less than $20 \%$ of the national emission limit of $20 \%$ to $30 \%$, can significantly reduce the original vehicle particulate emissions. The main reason is both of DOC and POC, respectively, can effectively reduce the soluble organic components SOF and carbon particles soot ${ }^{[3]}$ in tail gas particles. The POC filters the particle traps for specific analysis.

\section{PM/PN concentration distribution and POC filtration efficiency under different particle sizes}

The distribution of particulate matter and mass concentration distribution in different particle size under the hot and cold start conditions are analyzed. It is found that the activation state and POC had little effect on the particle size distribution of the particles. Therefore, in NEDC cycle, particles 
number and mass concentration of downstream of the POC in the total concentration are analyzed in the distribution of the particles at all levels.

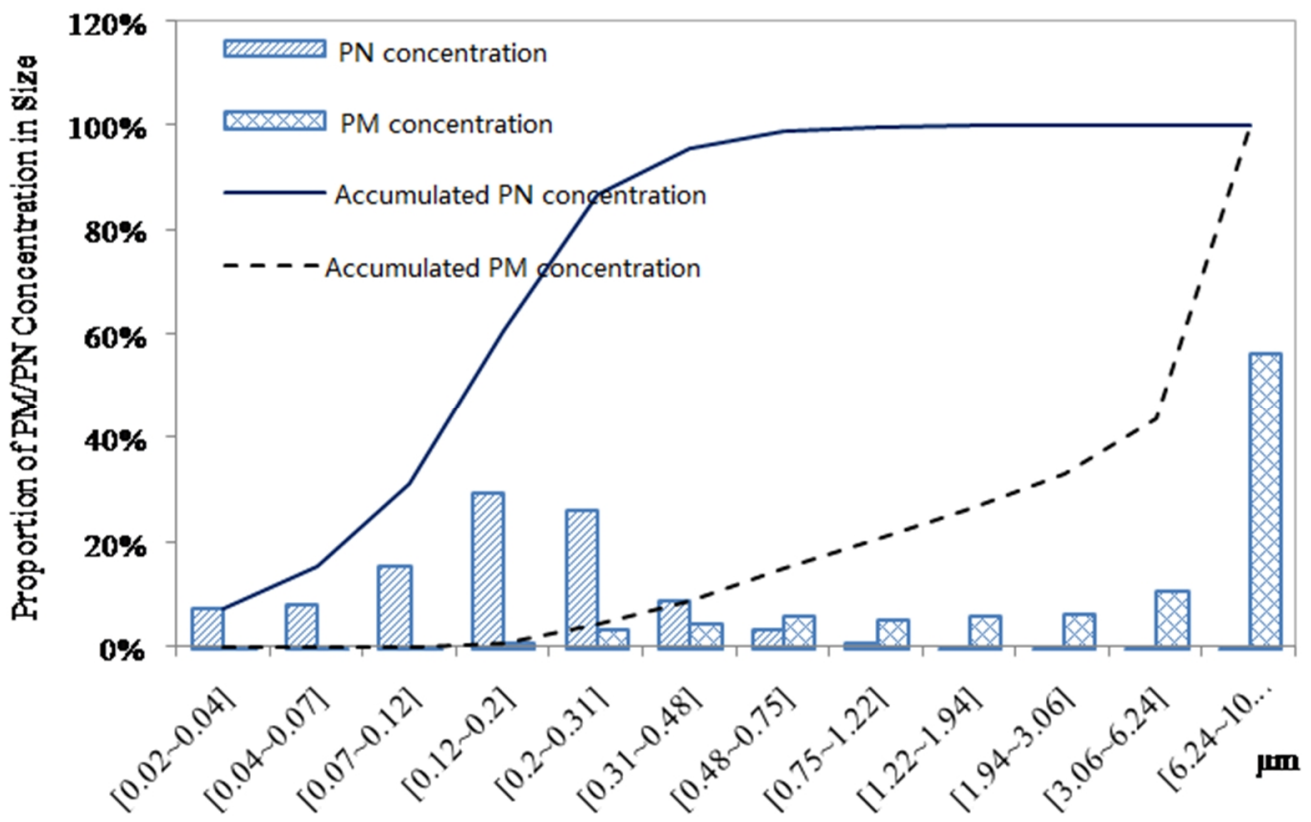

Fig.3 PM/PN distribution and POC filtration efficiency under different particle sizes

It can be seen from Figure 3, the particles number of the particle size less than $0.75 \mu \mathrm{m}$ in the total concentration of $99.71 \%$, the mass concentration of the total mass concentration is $20.48 \%$. Besides, the total concentration of particulate matter of particle size in $6.24 \sim 10.00 \mu \mathrm{m}$ is $0.01 \%$, and mass concentration of total mass concentration is $56.24 \%$. Therefore, the amount of diesel particulate matter emissions mainly come from fine particulate $\mathrm{PM}_{2.5}$, and particulate matter emissions mainly come from the particle size of $6.24 \sim 10.00 \mu \mathrm{m}$ coarse particles.

Figure 4 shows the distribution characteristics and filtration efficiency of the POC particles in different particle diameters under cold start and hot start conditions.

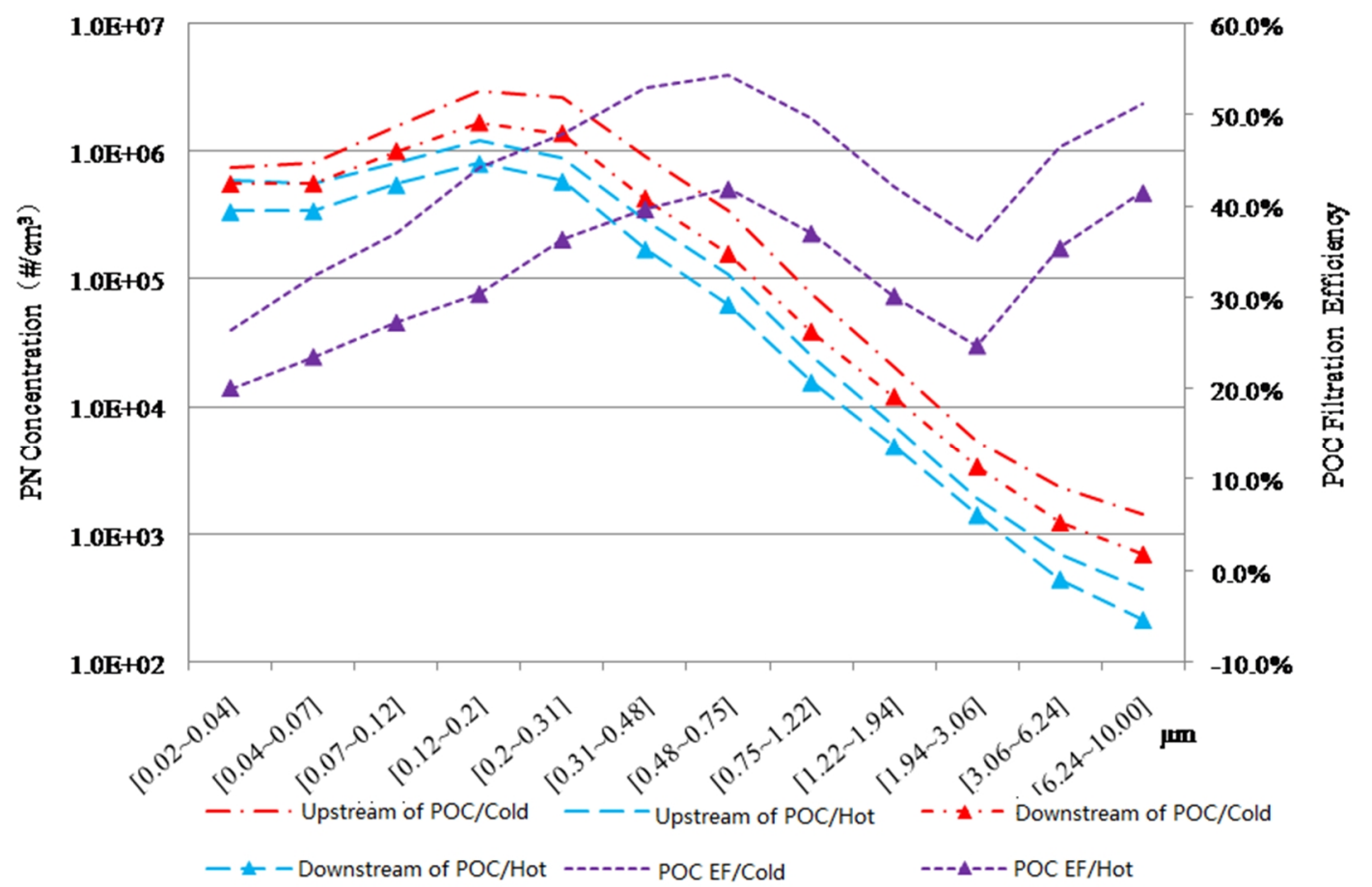

Fig.4 PN Distribution of Upstream and Downstream of POC under cold start and hot start condition 
It can be seen from Fig. 4, the particulate matter of upstream and downstream of POC present a single peak distribution, and the number of particles in the same trend of change in the particle size of $0.12 \sim 0.31 \mu \mathrm{m}$ peak $10^{+6} \mathrm{\#} / \mathrm{cm}^{3}$. Meanwhile, the amount of particulate matter concentration of upstream and downstream of POC is mainly concentrated in the particle size of $0.02 \sim 0.48 \mu \mathrm{m}$ fine particles. The amount of particulate matter in the upstream and downstream of POC under cold start is higher, mainly including the two reasons: (1) the low flow rate, low temperature, thicker and incomplete combustion, resulting in increased particulate emissions ${ }^{[4]}$; (2) DOC and POC carrier temperature is low under cold start, low efficiency of DOC $\mathrm{NO}$ into $\mathrm{NO}_{2}$, and POC does not meet the $\mathrm{NO}_{2}$ and carbon particles reaction temperature, resulting in carbon particles can not be efficient reaction regeneration and increasing particulate emissions.

Fig. 5 shows the distribution characteristics and filtration efficiency of the POC particles at different particle diameters under cold start and hot start.

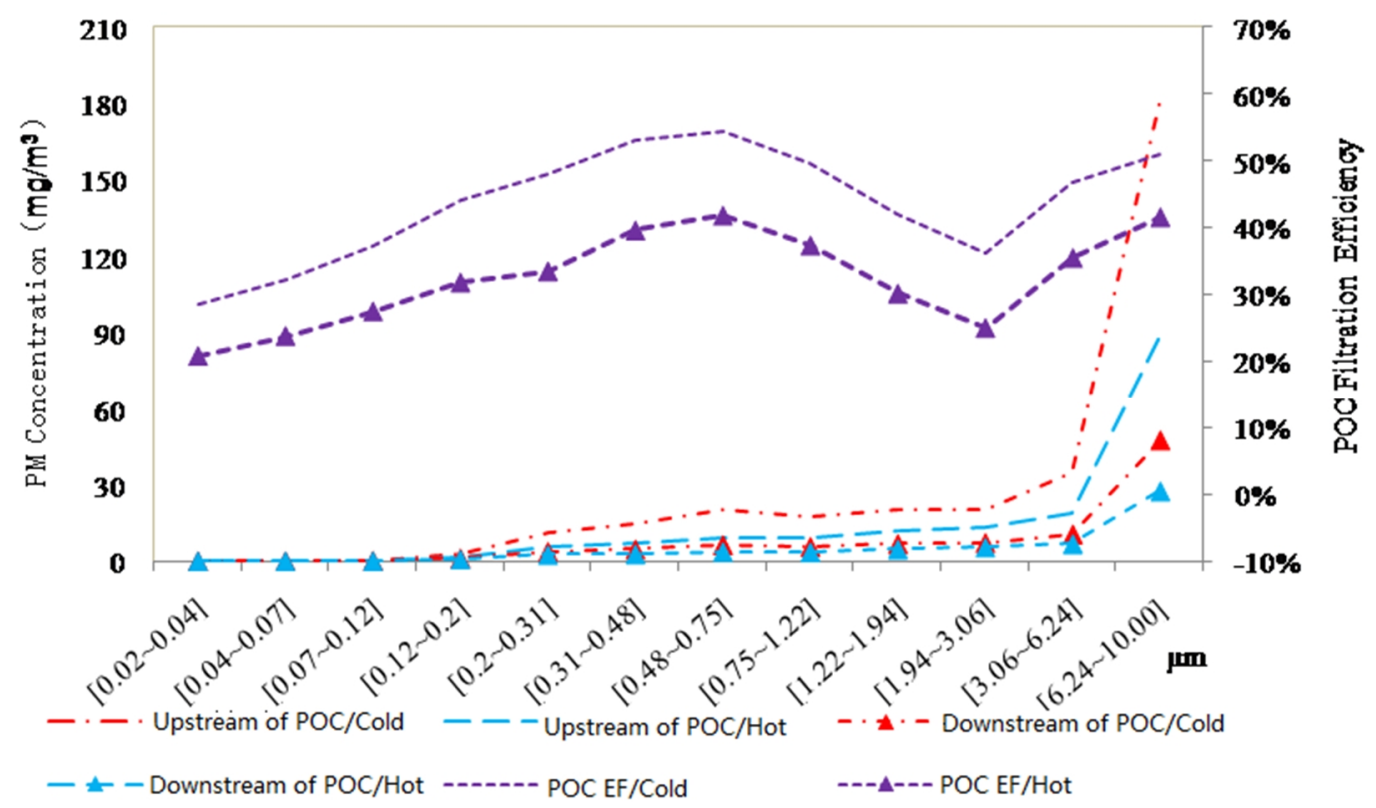

Fig.5 PM Distribution of Upstream and Downstream of POC under cold start and hot start condition

Fig.5 show that the particulate matter of upstream and downstream of POC particulate matter concentration with the particle size present a monotonically increasing trend in the particle size of $0.02 \sim 6.24 \mu \mathrm{m}$ within the particle concentration changes in the $6.24 \sim 10.00 \mathrm{~m}$ in the range of $180 \mathrm{mg} / \mathrm{m}^{3}$ and $90 \mathrm{mg} / \mathrm{m}^{3}$. The particles mass concentration of upstream and downstream of POC is mainly concentrated in the particle size of $6.24 \sim 10.00 \mu \mathrm{m}$. The mass concentration of particulate matter in upstream and downstream of the POC under cold start was higher than hot start, and the main reason was the same as the above concentration.

Compared with Fig.4 and Fig.5, the mass concentration of the filtration efficiency of POC in the particle size of the particles under cold and hot start contiditons is different, but the overall trend of change and POC filtration efficiency remained at 25\% 55\%. The POC filtration efficiency of the vehicle is about $10 \%$ higher than that of the hot start at the time of cold start. The possible reason for the analysis is that the exhaust temperature is low in the cold start, which is not conducive to the conversion of sulfur into the sulfur in the fuel, and the high exhaust temperature Easy to form sulfate, and easy to attach to the POC carrier, is not conducive to the exhaust carbon particles in the POC reaction, by reducing the hot start of the filtration efficiency.

For particle of size less than $0.75 \mu \mathrm{m}$, the POC filtration efficiency decreases monotonically with the decrease of the particle size. And the particle of size more than $0.75 \mu \mathrm{m}$, the POC filtration 
efficiency increases with the increase of the particle size. The reason is that when the particle size is less than $0.75 \mu \mathrm{m}$, the larger the particle size increase the larger the surface area, the particle is easier to be adsorbed and fixed on the surface of the POC carrier, and the finer particle surface area is small and the motion inertia is small, even if the particle size is less than $0.75 \mu \mathrm{m}$. When the particle size is more than $0.75 \mu \mathrm{m}$, the particle inertia increases with the increase of the particle size of the particles, and more particles follow the exhaust gas flow. When the particle size is larger than $0.75 \mu \mathrm{m}$, will be directly through the POC channel. For the coarse particles with particle size of 3.06 $\sim 10 \mu \mathrm{m}$, the porosity of the POC carrier determines that the coarse particles in the exhaust gas will be directly adsorbed on the surface of the carrier, and the filtration efficiency of the coarse particles will be high.

\section{Relationship between PM/PN Concentration and Speed}

Fig. 6 and Fig.7 for the particles number and mass concentration in upstream and downstream of $\mathrm{DOC}+\mathrm{POC}$ in NEDC cycle under cold and hot start conditions.

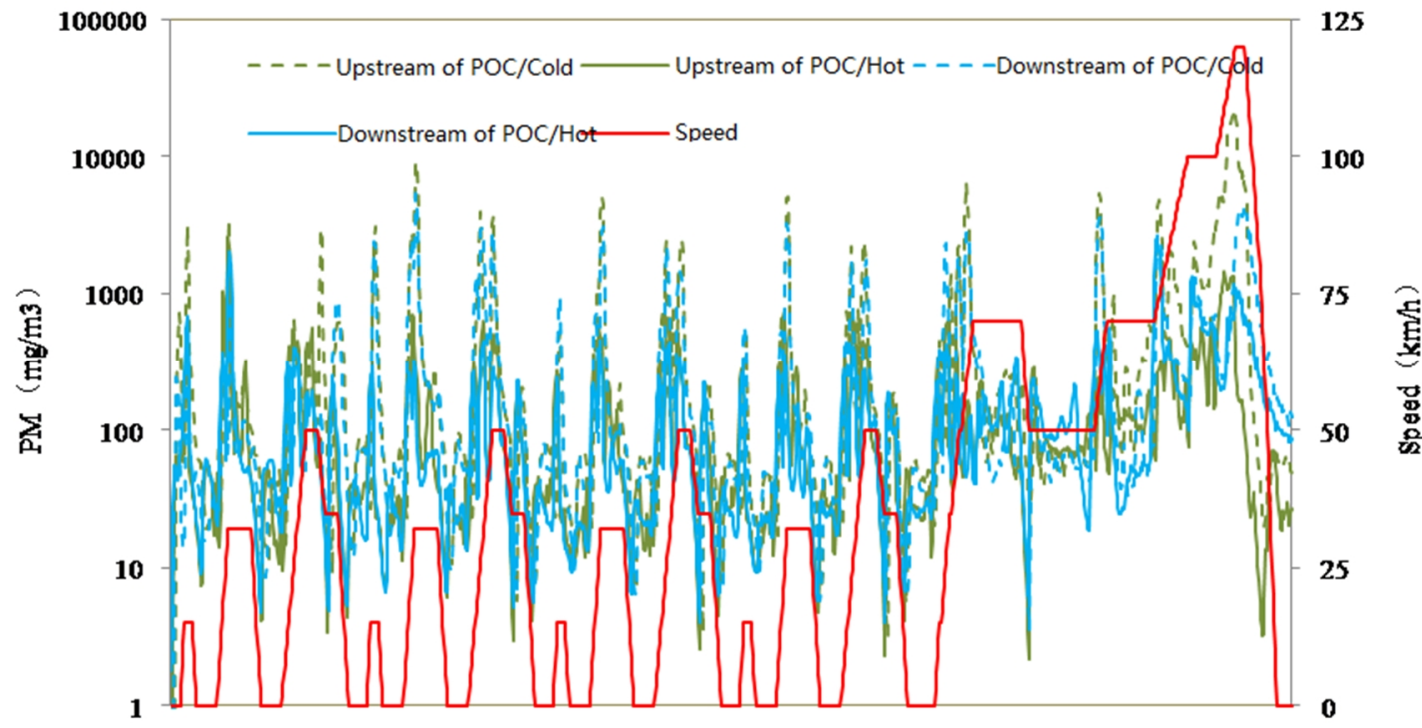

Fig.6 Relationship between PM Concentration and Speed

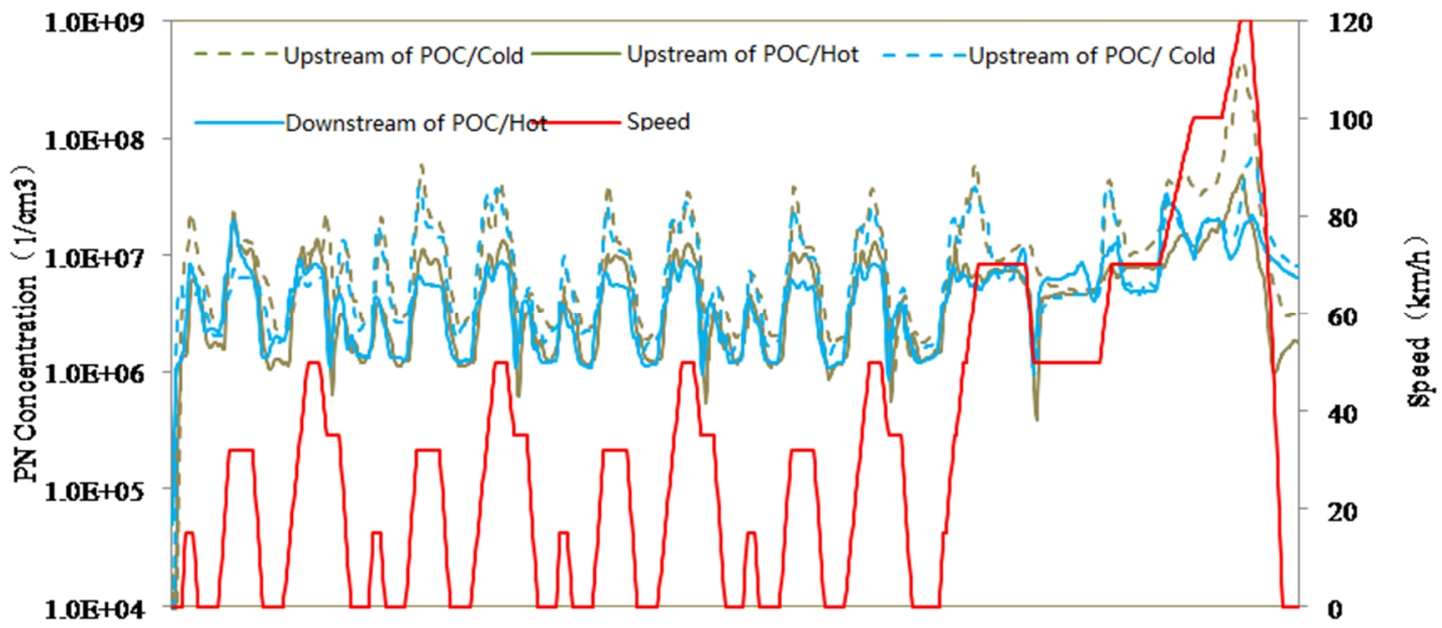

Fig.7 Relationship between PN Concentration and Speed

It can be seen from Fig. 6 and Fig. 7 that under the hot and cold start, the number concentration and mass concentration of the upstream and downstream of POC are consistent with the change of vehicle speed, and the particles are mainly produced in the acceleration condition, and the particles produced during deceleration and uniform velocity The main reason is that in the acceleration process, with the increase of the load, the temperature inside the cylinder gradually increased, the 
excess air coefficient becomes smaller, the mixture of local over-thickening situation, which constitutes a high temperature hypoxia, fuel crack The favorable conditions for the desorption of hydrogen are generated, resulting in a large number of soot cores which are subjected to processes such as surface growth and agglomeration, resulting in the formation of particulate matter.

\section{Conclusions}

(1) After the installation of DOC + POC under hot and cold start conditions, PM can meet the requirements of the four national emission limits, but has little effect on $\mathrm{NOx}$ and $\mathrm{HC}+\mathrm{NOx}$ emissions;

(2) The hot and cold starting mode and POC have little effect on the particle size distribution of particulate matter. The particulate matter emission from diesel vehicles is mainly from $\mathrm{PM}_{2.5}$, and the mass discharge is mainly from coarse particles in particle size of $6.24 \sim 10.00 \mu \mathrm{m}$;

(3) Under the cold start and hot start conditions, POC has different filtration efficiency of number concentration and mass concentration in each particle size, but the overall POC filtration efficiency is kept between $25 \%$ and $55 \%$. POC is mainly composed of $0.48 \sim 1.22 \mu \mathrm{m}$ Particulate matter and particle size of $6.24 \sim 10.00 \mu \mathrm{m}$ coarse particles filter effect is obvious, cold start POC filtration efficiency is higher than the hot start when about $10 \%$;

(4) The mass and number concentration of the particles before and after the POC are consistent with the trend of the vehicle speed. The particles are mainly produced in the acceleration condition, but the particles produced during the deceleration and uniform velocity are less.

\section{References}

[1] Li Shuhui. POC-an Aftertreatment Plan for Light-duty Diesel Vehicle GB III/GB IV[J]. Engine, 2008 (6): $: 40-46$.

[2] Gao Jidong, Qin Kongjian, Liang Rongliang. An Experimental Study on the PM and NOx Emission Characteristic of Public Bus in Real Driving Condition in Beijing. Automotive Engineering, 2011 (33) :757-760.

[3] Gao Xinwen. Study on the application of DOC+POC in the diesel engine with a straight line pump [D]. Master Thesis, Wuhan University of Technology.

[4] Wang Fengbin, Bao Junjiang, Qiao Weigao. An Experimental Study on the Particulate Emission from of Gasoline Vehicle Under Cycle Test Conditions[J]. Automotive Engineering, 2009 (31) :737-740. 